\title{
Direct Numerical Simulation and Large Eddy Simulation on a Turbulent Wall-Bounded Flow Using Lattice Boltzmann Method and Multiple GPUs
}

\author{
Xian Wang, ${ }^{1,2}$ Yanqin Shangguan, ${ }^{1}$ Naoyuki Onodera, ${ }^{2}$ \\ Hiromichi Kobayashi, ${ }^{3}$ and Takayuki Aoki ${ }^{2}$ \\ ${ }^{1}$ State Key Laboratory for Strength and Vibration of Mechanical Structures, School of Aerospace, Xian Jiaotong University, \\ 28 Xianning West Road, Xian 710049, China \\ ${ }^{2}$ Global Scientific Information and Computing Center, Tokyo Institute of Technology, 2-12-1 Ookayama, Meguro-ku, \\ Tokyo 152-8550, Japan \\ ${ }^{3}$ Department of Physics, Keio University, 4-1-1 Hiyoshi, Kouhoku-ku, Yokohama 223-8521, Japan
}

Correspondence should be addressed to Xian Wang; wangxian@mail.xjtu.edu.cn

Received 4 January 2014; Accepted 11 February 2014; Published 14 April 2014

Academic Editor: Gongnan Xie

Copyright (C) 2014 Xian Wang et al. This is an open access article distributed under the Creative Commons Attribution License, which permits unrestricted use, distribution, and reproduction in any medium, provided the original work is properly cited.

\begin{abstract}
Direct numerical simulation (DNS) and large eddy simulation (LES) were performed on the wall-bounded flow at $\mathrm{Re}_{\tau}=180$ using lattice Boltzmann method (LBM) and multiple GPUs (Graphic Processing Units). In the DNS, 8 K20M GPUs were adopted. The maximum number of meshes is $6.7 \times 10^{7}$, which results in the nondimensional mesh size of $\Delta^{+}=1.41$ for the whole solution domain. It took 24 hours for GPU-LBM solver to simulate $3 \times 10^{6} \mathrm{LBM}$ steps. The aspect ratio of resolution domain was tested to obtain accurate results for DNS. As a result, both the mean velocity and turbulent variables, such as Reynolds stress and velocity fluctuations, perfectly agree with the results of Kim et al. (1987) when the aspect ratios in streamwise and spanwise directions are 8 and 2, respectively. As for the LES, the local grid refinement technique was tested and then used. Using $1.76 \times 10^{6}$ grids and Smagorinsky constant $\left(C_{s}\right)=0.13$, good results were obtained. The ability and validity of LBM on simulating turbulent flow were verified.
\end{abstract}

\section{Introduction}

Lattice Boltzmann method has been regarded as a promising alternative for simulation of fluid flows with complex physics in the past two decades. Unlike conventional numerical schemes based on discretizations of macroscopic continuum equations, the LBM is based on microscopic models and mesoscopic kinetic equations. It has many advantages, including easy implementation of boundary conditions, easy programming, and fully parallel algorithms [1]. As a result, the LBM has been applied in many fields, such as biofluid and porous medium [2]. But its feasibility and validity in both direct numerical simulation and large eddy simulation of turbulent flow remain to need further validation $[3,4]$.

Fully developed turbulent wall-bounded flow is a classic problem, which is seemingly simple but complicated.
The DNS and LES on it, including the boundary layer flow, have become important tools for investigating the mechanism of turbulence. For a long time, the simple geometry attracts many experimental and theoretical investigations of complex turbulence interactions in the vicinity of walls. Among them the direct numerical simulation done by Kim et al. $[5,6]$ is most cited for comparison. Their DNS results of $\operatorname{Re}_{\tau}=u_{\tau} \delta / v=180\left(u_{\tau}\right.$ and $\delta$ are the friction velocity and channel half-width, resp.,) have been considered as the benchmark of numerical simulations. Because of the large computational cost of DNS and LES, the published related results on wall-bounded flow are rare in spite of the increasingly high performance of high performance computing (HPC) in the recent decade years.

Nowadays, the presence of GPU brings possibility to the large-scaled DNS and LES for turbulence prediction. GPU 
equipped on the graphic board in a computer is originally used to process large graphics data sets fast. Recently, with the emergence and developing of computing platforms, for example, CUDA [7] and OpenCL, the use of GPU to accelerate nongraphic computations has drawn more and more attention $[8,9]$. Due to its high performance of floating-point arithmetic operation, wide memory bandwidth, and better programmability, GPU has been further used for the general purposes, that is, GPGPU. Computational fluid dynamics (CFD) based on grids is one of the important applications. According to our experience, solving incompressible NavierStokes equations to simulate fluid flow with the marker and cell (MAC) solver on single GPU is $30 \sim 40$ times faster than the heavily optimized CPU-based implementations. And the LBE GPU-based calculations can even obtain more than 100 speedups [10-13]. Aoki group [14] has devoted to the GPU high-performance computing since 2006. The team members performed various numerical simulations using hundreds or even thousands of GPUs, such as weather prediction, crystal growth process, and air flow in the city, and a good performance of PFLOPS was achieved [15-18]. At present, GPU applications for engineering purpose in fields such as energy, environment, and aerospace become hotspots.

In the present work, DNS and LES on the wall-bounded flow at $\mathrm{Re}_{\tau}=180$ were performed using D3Q19 model of lattice Boltzmann method and multiple GPUs. There are two objectives. One is to present a high performance computing with LBM and multi-GPUs, which may provide a possible way for the prediction on turbulent flow. The other is to verify the validity and ability of LBM on simulating turbulent flow.

This paper is organized as follows. In Section 2, lattice Boltzmann equations (LBE) for DNS and LES with forcing term are described. Section 3 introduces the model system of the simulation and data transfer in CUDA-MPI. The results of DNS and LES on fully developed turbulent wall-bounded flow are discussed in Sections 4 and 5, respectively. The summary and conclusions are presented in Section 6.

\section{Lattice Boltzmann Equation (LBE) Formulation for DNS and LES of Turbulence}

2.1. LBE for DNS. In LBM, firstly, the following Boltzmann equation for the discrete velocity distribution is solved on a discrete lattice [19]:

$$
\frac{\partial f_{i}}{\partial t}+\mathbf{e}_{i} \cdot \nabla f_{i}=\Omega_{i} \quad(i=1,2, \ldots, N)
$$

where $f_{i}$ is the particle velocity distribution function, $\mathbf{e}_{i}$ is the particle velocity in the $i$ th direction, and $N$ is the number of velocities. For LBM, the way of classifying the different methods by lattice is the DnQm scheme. Here " $D n$ " stands for " $n$ dimensions," while " $Q m$ " stands for " $m$ speeds." For $2 \mathrm{D}$ model, 9-velocity LBM is the most common. For 3D model, there are several cubic lattice models, such as D3Q13, D3q15,
D3Q19, and D3Q27 ( $N=13,15,19$, or 27$). \Omega_{i}$ is the collision operator. Using Boltzmann-BGK approximation [20],

$$
\Omega_{i}=-\frac{1}{\tau}\left(f_{i}-f_{i}^{\mathrm{eq}}\right),
$$

we get

$$
\frac{\partial f_{i}}{\partial t}+\mathbf{e}_{i} \cdot \nabla f_{i}=-\frac{1}{\tau}\left(f_{i}-f_{i}^{\mathrm{eq}}\right),
$$

where $f_{i}^{\text {eq }}$ is the local equilibrium distribution and $\tau$ is the relaxation time. To obtain the Navier-Stokes equations, the equilibrium distribution functional form must be carefully chosen. In the 9-speed square lattice and the 13-, 15-, 19-, and 27-speed cubic lattices, a suitable equilibrium distribution function has been proposed as [2]

$$
f_{i}^{\mathrm{eq}}=\rho \omega_{i}\left[1+3 \mathbf{e}_{i} \cdot \mathbf{u}+\frac{9}{2}\left(\mathbf{e}_{i} \cdot \mathbf{u}\right)^{2}-\frac{3}{2} \mathbf{u} \cdot \mathbf{u}\right] .
$$

In the present study, D3Q19 model was adopted with $e_{0}(0,0$, $0), e_{1}(1,0,0), e_{2}(-1,0,0), e_{3}(0,1,0), e_{4}(0,-1,0), e_{5}(0,0,1)$, $e_{6}(0,0,-1), e_{7}(1,1,0), e_{8}(-1,1,0), e_{9}(1,-1,0), e_{10}(-1,-1,0)$, $e_{11}(1,0,1), e_{12}(-1,0,1), e_{13}(1,0,-1), e_{14}(-1,0,-1), e_{15}(0,1$, $1), e_{16}(0,-1,1), e_{17}(0,1,-1)$, and $e_{18}(0,-1,-1)$. Figure 1 shows the D3Q19 model we used here. The corresponding weighting factors are $\omega_{0}=1 / 3, \omega_{1} \sim \omega_{6}=1 / 18$, and $\omega_{7} \sim \omega_{18}=1 / 36$. $\rho$ and $\mathbf{u}$ are the macroscopic quantities and can be evaluated as [2]

$$
\begin{gathered}
\rho=\sum_{i=0}^{N} f_{i}=\sum_{i=0}^{N} f_{i}^{\mathrm{eq}}, \\
\rho \mathbf{u}=\sum_{i=0}^{N} f_{i} \mathbf{e}_{i}=\sum_{i=0}^{N} f_{i}^{\mathrm{eq}} \mathbf{e}_{i} .
\end{gathered}
$$

The speed of sound is $c_{s}=1 / \sqrt{3}$, and the equation of state is that of an ideal gas as follows:

$$
p=\rho c_{s}^{2}=\frac{\rho}{3} .
$$

Equation (3) can be further discretized in physical space $\mathbf{x}$ and time $t$. The completely discretized form of (3) is

$$
f_{i}\left(\mathbf{x}+\mathbf{e}_{i} \delta t, t+\delta t\right)-f_{i}(\mathbf{x}, t)=-\frac{1}{\tau}\left[f_{i}(\mathbf{x}, t)-f_{i}^{\mathrm{eq}}(\mathbf{x}, t)\right] .
$$

Equation (7) is the well-known LBGK model, where $\tau=\lambda / \delta t$ is the nondimensional relaxation time. The viscosity in the macroscopic Navier-Stokes equation can be derived from (7) as

$$
v=\left(\tau-\frac{1}{2}\right) \delta t
$$

Equation (7) is usually solved with its standard form by assuming that $\delta t=1$ according to the following two steps.

Collision step:

$$
\tilde{f}_{i}(\mathbf{x}, t)=f_{i}(\mathbf{x}, t)-\frac{1}{\tau}\left[f_{i}(\mathbf{x}, t)-f_{i}^{\mathrm{eq}}(\mathbf{x}, t)\right] .
$$



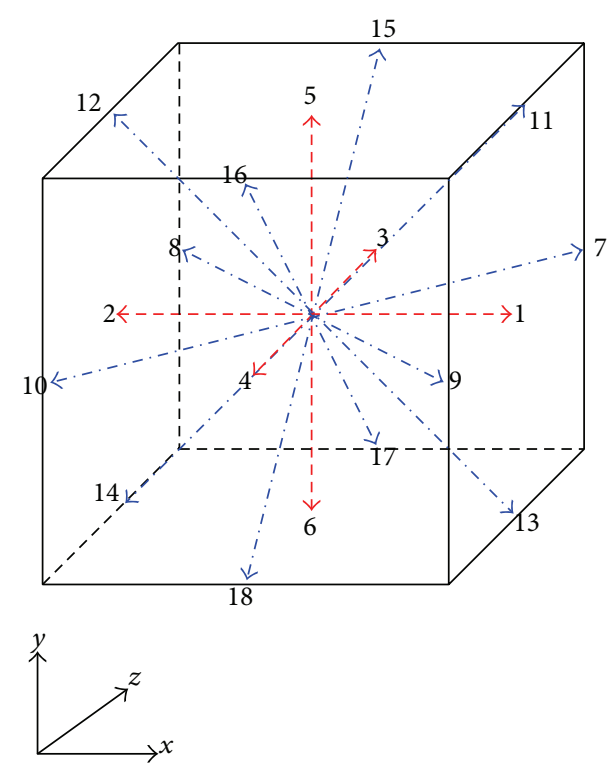

FIGURE 1: D3Q19 LBM model.

Streaming step:

$$
f_{i}\left(\mathbf{x}+\mathbf{e}_{i}, t+1\right)=\widetilde{f}_{i}(\mathbf{x}, t),
$$

where $f_{i}$ and $\tilde{f}_{i}$ denote the pre- and postcollision states of the distribution function, respectively.

2.2. LBE for LES. The filtered form of the LBE for LES is defined as [21-23]

$$
\begin{aligned}
\bar{f}_{i}\left(\mathbf{x}+\mathbf{e}_{i} \delta_{t}, t+\delta_{t}\right) & =\bar{f}_{i}\left(\mathbf{x}, \delta_{t}\right)-\frac{1}{\tau_{*}}\left[\bar{f}_{i}-\bar{f}_{i}^{\mathrm{eq}}\right], \\
\tau_{*} & =\tau_{0}+\tau_{t},
\end{aligned}
$$

where $\bar{f}_{i}$ and $\bar{f}_{i}^{\mathrm{eq}}$ represent the distribution function and the equilibrium distribution function of the resolved scales, respectively. Moreover, $\tau_{0}$ and $\tau_{t}$ are the relaxation time corresponding to the molecular viscosity $v_{0}$ and eddy viscosity $v_{t}$, respectively. Accordingly, $v_{*}$ is given by [21-23]

$$
\begin{aligned}
& v_{*}=v_{0}+v_{t}=\frac{1}{3}\left(\tau_{*}-\frac{1}{2}\right) c^{2} \delta_{t}, \\
& v_{0}=\frac{1}{3}\left(\tau_{0}-\frac{1}{2}\right) c^{2} \delta_{t}, \quad v_{t}=\frac{1}{3} \tau_{t} c^{2} \delta_{t} .
\end{aligned}
$$

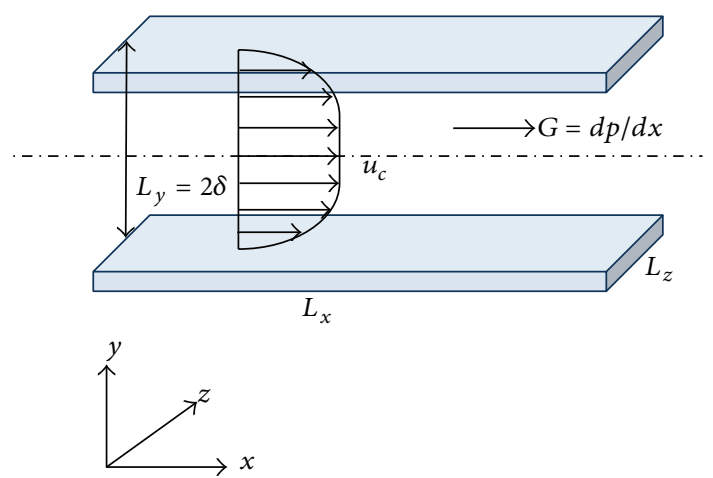

Figure 2: Model system.

The eddy viscosity $v_{t}$ arising from the standard Smagorinsky model can be calculated with the filter length scale $\Delta_{x}$ and the filtered strain rater tensor $\bar{S}_{i j}=\left(\partial_{j} \bar{u}_{i}+\partial_{i} \bar{u}_{j}\right) / 2$ as [23]

$$
v_{t}=\left(C_{s} \Delta_{x}\right)^{2} \overline{\mathrm{S}}, \quad \overline{\mathrm{S}}=\sqrt{2 \sum_{i j} \overline{\mathrm{S}}_{i j} \overline{\mathrm{S}}_{i j}}
$$

Here, $C_{s}$ is Smagorinsky constant. In addition, finitedifference approximation was chosen to compute the strain rater tensor in the present work.

2.3. External Force. For the external forcing term, there are many implementation methods. In the presented wallbounded flow simulation, the modified velocity method [24] was applied that is using a modified velocity to compute the equilibrium distribution function. It can be described as follows:

$$
\rho \mathbf{u}^{\mathrm{eq}}=\rho \mathbf{u}+\tau \mathbf{G},
$$

where $\mathbf{G}$ is the external force. As the wall-bounded flow simulated here is driven by a constant body force, the uniform pressure drop, we define $\mathbf{G}$ as

$$
\mathbf{G}=\frac{d p}{d x}=\frac{u_{\tau}^{2} \rho}{\delta} .
$$

Here, $u_{\tau}$ and $\delta$ represent the friction velocity and channel half-width, respectively.

Then calculate the equilibrium distribution function $f_{i}^{\text {eq }}$ with $\mathbf{u}^{\mathrm{eq}}$ instead of $\mathbf{u}$. So (4) can be transferred as

$$
f_{i}^{\mathrm{eq}}=\rho \varrho_{i}\left[1+3 \mathbf{e}_{i} \cdot \mathbf{u}^{\mathrm{eq}}+\frac{9}{2}\left(\mathbf{e}_{i} \cdot \mathbf{u}^{\mathrm{eq}}\right)^{2}-\frac{3}{2} \mathbf{u}^{\mathrm{eq}} \cdot \mathbf{u}^{\mathrm{eq}}\right] .
$$

2.4. Boundary Conditions. As for the boundary conditions of $\tilde{f}$, periodic boundary condition was used in streamwise and spanwise direction of the present work. For the walls, the following boundary condition was applied:

$$
\begin{aligned}
\tilde{f}_{i} & =f_{i}^{\text {eq }}+f_{i}^{\text {neq }}=f_{i}^{\text {eq }}+f_{i \text { neighbor }}^{\text {neq }} \\
& =f_{i}^{\text {eq }}+\left(\tilde{f}_{i \text { neighbor }}-f_{i \text { neighbor }}^{\text {eq }}\right),
\end{aligned}
$$




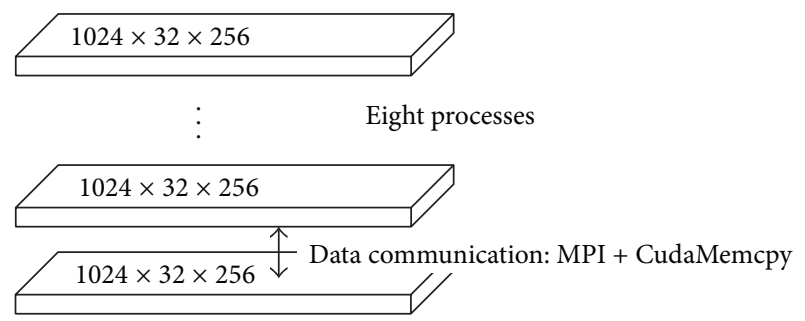

FIGURE 3: 1D partitioning in $y$-direction.

where $f_{i}^{\text {eq }}$ was computed by the macroscopic values $\mathbf{u}$ and $\rho$ on the boundaries according to (4). $f_{i}^{\text {neq }}$ cannot be computed directly and was assumed to be equal to the value of its neighboring inner node. $\tilde{f}_{i \_ \text {neighbor }}$ and $f_{i \_ \text {neighbor }}^{\text {eq }}$ were computed by (9) and (4), respectively, with the corresponding $\mathbf{u}$ and $\rho$.

\section{Model System and Parameters}

Figure 2 shows the model system. The calculation domain length is $L x, L y$, and $L z$ corresponding to the streamwise $x$, normal $y$, and spanwise $z$ direction, respectively. $\delta$ is halfwidth in the normal direction to walls, which is used to define the Reynolds number. The standard Reynolds number can be given by $\operatorname{Re}_{c}=\delta u_{c} / v$ with the mean centerline velocity. In addition, it is common to define the Reynolds number in such flow model using the "wall unit" or viscous length $l_{\tau}=v / u_{\tau}$. The skin friction is calculated as $u_{\tau}=\sqrt{\mathbf{G} \delta / \rho}$ and the corresponding "wall unit" time is $t_{\tau}=l_{\tau} / u_{\tau}$. Whereby, the friction Reynolds number is obtained as

$$
\operatorname{Re}_{\tau}=\frac{\delta u_{\tau}}{v},
$$

which implies $\operatorname{Re}_{\tau}=\delta / l_{\tau}=\delta^{+}$. As usual, a superscript (+) indicates quantities measured in the "wall units" $l_{\tau}$ and $u_{\tau}$. In a fully developed turbulent wall-bounded flow beyond the transient range of Reynolds numbers, $\operatorname{Re}_{c} \geq \operatorname{Re}_{\tau}$.

To carry out numerical simulation of turbulence, the nondimensional physical parameters describing the problem must be defined first. For wall-bounded flow, the spatial extent of the computational domain plays an important role to obtain accurate results. In particular, the aspect ratios are $\alpha_{x}=L_{x} / \delta$ and $\alpha_{z}=L_{z} / \delta$. Referring to the study in [26], Spasov et al. [27] performed the direct numerical simulations of $\operatorname{Re}_{\tau}=180$ at $\alpha_{x}=4$ and $\alpha_{z}=1$. The mean velocities were overpredicted compared with the results obtained by Kim et al. [5]. Also, the Reynolds stress did not agree well. In Section 4, we verified various groups of aspect ratio to perform DNS.

For DNS, the grid size cannot be larger than the local Kolmogorov length $\eta$. In fully developed turbulent flow, $\eta^{+}=$ 1.5 in the region near the wall [28]. $\eta^{+}$increases with the increasing distance from the wall. Owing to the uniform mesh used in LBM, the grid scale in the whole field should satisfy $\Delta^{+}<\eta^{+}=1.5$. As for the LBM, $\Delta=1, \Delta^{+}=\Delta / l_{\tau}=$ $\operatorname{Re}_{\tau} / \delta<1.5$, which results in $\delta>\operatorname{Re}_{\tau} / 1.5$. Accordingly, with the friction Reynolds number in the present work $\operatorname{Re}_{\tau}=180$, the half-width $\delta$ in the computational domain should be large than 120.

As for initial conditions, $\rho=\rho_{0}=1$ and $u_{\tau}$ was given by logarithmic law of friction: $u_{c} / u_{\tau}=(1 / \kappa) \ln \operatorname{Re}_{\tau}+b$, in which von Karman constant $\kappa=0.4, b=6$, and $u_{c}$ was set as 0.1. The velocity field was initialized as follows: $u=\bar{u}+u^{\prime}, v=\bar{v}+v^{\prime}$, and $w=\bar{w}+w^{\prime}$, where the fluctuating terms were got from $u^{\prime}=v^{\prime}=w^{\prime}=u_{c}\left(r_{\text {rand }}-0.5\right), r_{\text {rand }} \in[0,1], \bar{v}=\bar{w}=0$, and $\bar{u}$ was based on a frequently used engineering approximation for the universal mean velocity profile near the wall

$$
u^{+}= \begin{cases}y^{+}, & y^{+} \leq y_{w}, \\ \frac{\ln \left(y^{+}\right)}{\kappa+B}, & y^{+}>y_{w} .\end{cases}
$$

The dimensionless velocity $u^{+}=\bar{u} / u_{\tau}$ is measured in the "wall units." The particular values chosen, $y_{w}=11.6$ for the velocity profile inflection point, $\kappa=0.4$ for the Van Karman constant, and $B=5.2$, are not expected to have an impact on the eventual flow profile taken for the computation of developed turbulence statistics.

Current parallel computation was made by $1 \mathrm{D}$ domain partitioning using 8 GPUs. Figure 3 shows the $1 D$ partitioning in $y$-direction executed in the present study. The data are exchanged with top and bottom ranks. As for detailed information on data transfer, refer to our previous work [12]. Data communications among GPUs are done by Message Passing Interface (MPI) and cudaMemcpy. First, the data are copied from GPU to CPU by CUDA API cudaMemcpy(...,cudaMemcpyDeviceToHost). Then the data are exchanged between corresponding CPUs using parallel tool like MPI, and so forth. Finally, the exchanged data are copied from CPU to GPU by cudaMemcpy(...,cudaMemcpyHostToDevice).

\section{DNS for Wall-Bounded Flow}

The DNS was performed on turbulent wall-bounded flow with $\delta=128$ and various aspect ratios. The corresponding nondimensional mesh size is $\Delta^{+}=1.41$ for the whole solution domain. Figure 4 shows (a) the velocity pattern at a constant streamwise location and (b) the isosurface of second invariant of velocity gradient tensor $Q$ when the aspect ratio is $\alpha_{x}=4$, $\alpha_{z}=1$.

4.1. Effect of Aspect Ratio. Figure 5 shows the profile of averaged velocity in the normal $y$-direction at the aspect ratios (a) $\alpha_{x}=4, \alpha_{z}=1$, (b) $\alpha_{x}=4, \alpha_{z}=2$, (c) $\alpha_{x}=8, \alpha_{z}=$ 1 , and (d) $\alpha_{x}=8, \alpha_{z}=2$, in which our DNS results shown by solid lines and the dotted lines represent the DNS results got by Kim et al. [5]. It is obvious that the averaged velocity at $\alpha_{x}=8, \alpha_{z}=2$ fits perfectly with the result of Kim et al. Although the periodical boundary condition is used in spanwise direction, for the same dimension in streamwise direction, larger spanwise ratio makes the results match better by comparing Figure 5(a) with Figure 5(b) and comparing Figure 5(c) with Figure 5(d). However, the dimension in 


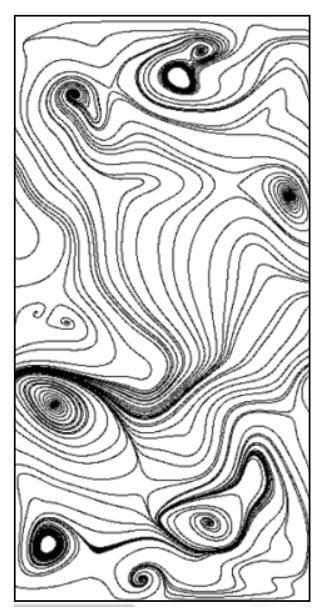

(a)

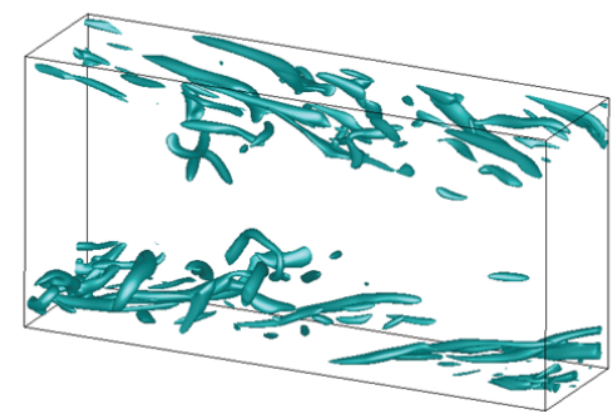

(b)

Figure 4: (a) Velocity pattern at a constant streamwise location, (b) isosurface of second invariant of velocity gradient tensor at $\alpha_{x}=4$, $\alpha_{z}=1$.

streamwise direction, that is, $\alpha_{x}$, has little effect on the mean velocity (comparing Figure 5(a) with Figure 5(c) and Figure 5(b) with Figure 5(d)).

Figure 6 presents the turbulent statistics calculated by LBM-DNS and comparison with the DNS data by Kim et al. [5] for (i) Reynolds stress in wall-normal directions, (ii) the components of the root-mean-square (rms) in streamwise, spanwise, and wall-normal velocity fluctuations, at (a) $\alpha_{x}=4$, $\alpha_{z}=2$, (b) $\alpha_{x}=8, \alpha_{z}=2$, respectively. It can be seen that our LBM-DNS results and Kim's data [5] are generally in good agreement for the two cases of aspect ratio. There are slight discrepancies of rms at $\alpha_{x}=4, \alpha_{z}=2$, especially the rms in streamwise velocity fluctuations indicated by u_rms. Comparing with mean velocity and Reynolds stresses, DNS results of velocity fluctuations are more sensitive to the streamwise length chosen for simulation.

4.2. Performance of Computation by GPUs. For this DNS work, it took about 24 hours to simulate $3 \times 10^{6}$ LBM-steps for $6.7 \times 10^{7} \mathrm{LBM}$-grids with $8 \mathrm{~K} 20 \mathrm{M}$ GPUs. The performance achieves 2333 MLUPS; that is, $2.333 \times 10^{9}$ meshes are processed per second. Compared with the similar DNS in [27] done by $36 \mathrm{CPUs}$, in which the performance is 5.6 MLUPS, the present performance by GPUs is about 416 times higher. With LBM-GPU, high performance can be achieved easily without any difficulty in programming.

\section{LES for Wall-Bounded Flow}

Since the DNS approach resolves all relevant spatial and temporal scales, it can predict all possible fluid motions with high fidelity but large cost. In this part, we simulated wallbounded flow by applying the standard Smagorinsky model to LES. Moreover, the local grid refinement technique of LBM was tested and then used.
5.1. Local Grid Refinement. Local grid refinement is usually applied to regions where large changes of solution are expected. For LBM, the locally refined patches are superposed to the global coarse grid, saving computational time to some extent and enabling a high resolution where needed. Grid refinement is performed by dividing the space step through a refinement factor $n$. The kinematic viscosity, defined in the frame of the LBGK model, depends on the step size with $v=((2 \tau-1) / 6) \delta t$. To keep a constant viscosity and the same Reynolds number on coarse grids $\left(\delta x_{c}\right)$ and fine grids $\left(\delta x_{f}=\delta x_{c} / n\right)$, the relaxation time $\tau$ in (8) has to be redefined as [29]

$$
\tau_{f}=\frac{1}{2}+n\left(\tau_{c}-\frac{1}{2}\right)
$$

Here $\tau_{f}$ and $\tau_{c}$ represent the relaxation time on the fine and coarse grids, respectively. Taking into account that when $1 / \tau_{c}$ is very close to 2 , the LBGK scheme becomes unstable and $1 / \tau_{f}>1$, we estimate from (20) that the upper limit of $n$ is about 50. In the present study the value of refinement factor $n$ is 2 . Accordingly, the time step on the fine grid is decreased by $\delta t_{f}=\delta t_{c} / n=\delta t_{c} / 2$, in which $\delta t_{c}$ is the time-step on the coarse grid. Because of the continuity of the hydrodynamic variables and their derivatives on the interface between two grids, the relationships between postcollision distribution functions on the coarse and fine grid nodes obtained from (4), (9), and (20) can be described as follows, respectively:

$$
\begin{aligned}
& \tilde{f}_{i}^{(c)}=f_{i}^{(\mathrm{eq}, f)}+n \frac{\tau_{c}-1}{\tau_{f}-1}\left[\widetilde{f}_{i}^{(f)}-f_{i}^{(\mathrm{eq}, f)}\right], \\
& \widetilde{f}_{i}^{(f)}=f_{i}^{(\mathrm{eq}, c)}+\frac{\tau_{f}-1}{\left(n\left(\tau_{c}-1\right)\right)}\left[\widetilde{f}_{i}^{(c)}-f_{i}^{(\mathrm{eq}, c)}\right] .
\end{aligned}
$$

Strategy of the numerical realization with refinement factor $n=2$ can be seen in Figure 7. Firstly, carry out a simulation on coarse grids to $t 1$. Then, map the solution to fine grid using second-order interpolation in space and time from the 


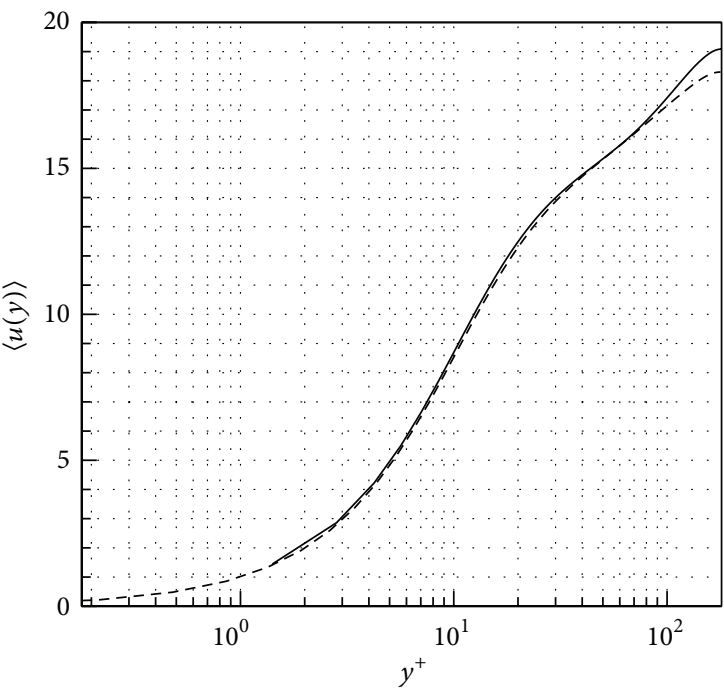

(a)

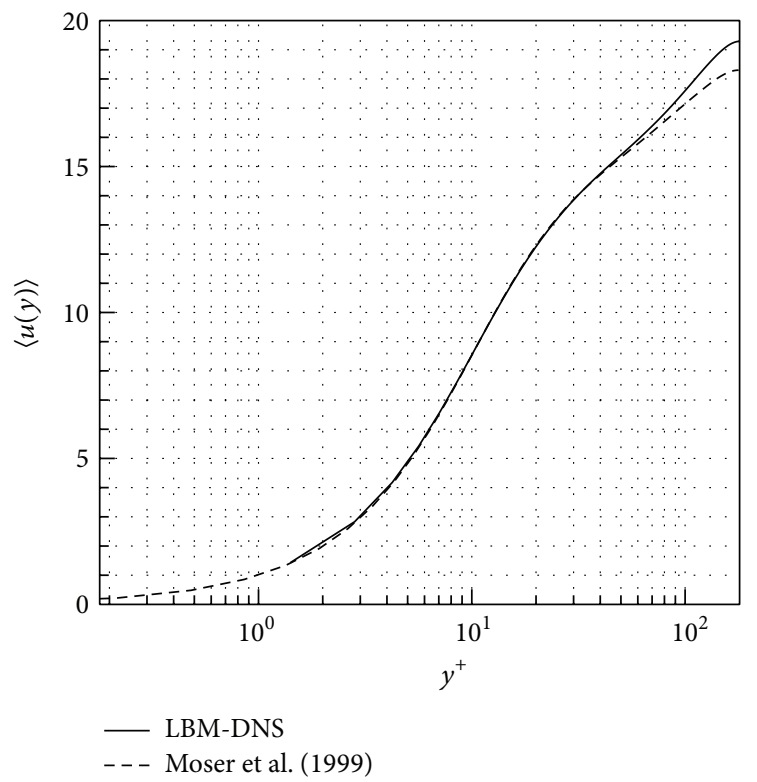

(c)

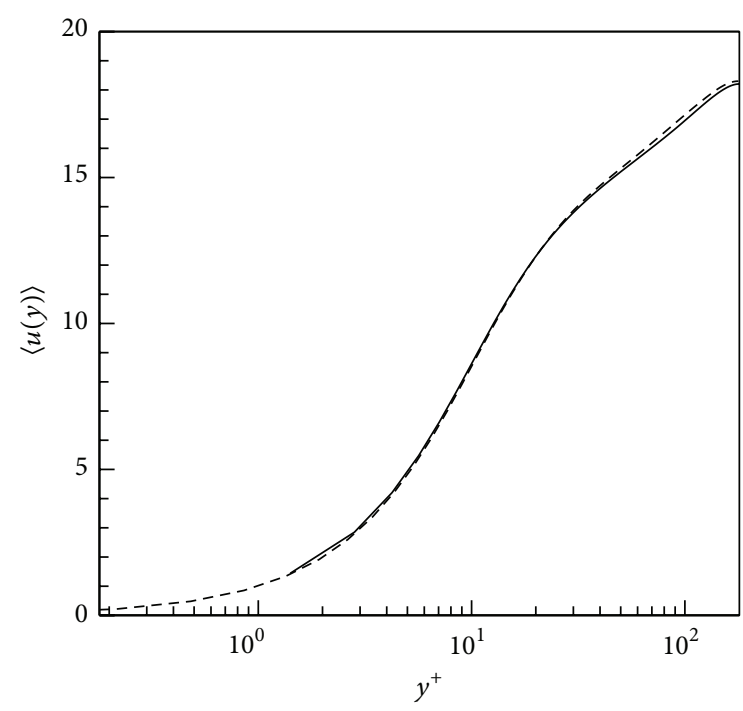

(b)



(d)

FIGURE 5: The profile of averaged velocity in the normal $y$-direction at (a) $\alpha_{x}=4, \alpha_{z}=1$, (b) $\alpha_{x}=4, \alpha_{z}=2$, (c) $\alpha_{x}=8$, $\alpha_{z}=1$, and (d) $\alpha_{x}=8, \alpha_{z}=2$.

results of coarse grids at $t$, according to (22). Finally, advance the dependent variables on the coarse and fine grids in time proceeding with the obtained fine grid boundary conditions.

The validity of the adopted local grid refinement was verified by simulating the classical problem of lid-driven cavity flow at $\operatorname{Re}=1000$ with and without local grid refinement. Local grid refinement was used with a coarse grid $(64 \times 32$ nodes $)$ in middle part and a fine grid $(16 \times 127$ nodes $)$ in 1/4 top and bottom computational region, respectively. The comparison between the NSE results of Ghia et al. [25] and our LBE results with and without using grid refinement on this problem is presented by velocity profile on the center line in the normal direction in Figure 8. It is clearly shown that the LBE results adopted grid refinement coincide better with the results gained by Ghia et al. [25] near boundaries where the grids are refined than the ones by the uniform coarse grid system.

5.2. Results and Discussion. For LES, first, the number of grids is decreased by 4 times in each direction based on the DNS and the aspect ratio is keep to $\alpha_{x}=8, \alpha_{z}=2$. That is, the total grids for LES is $1 / 64$ of DNS. Figure 9 displays the profile of averaged velocity in the normal $y$-direction using LBM-LES at various $C_{s}$ for a grid system $256 \times 64 \times 64$, whose grid scale is $\Delta^{+}=5.64$. The results at different $C_{s}$ are 

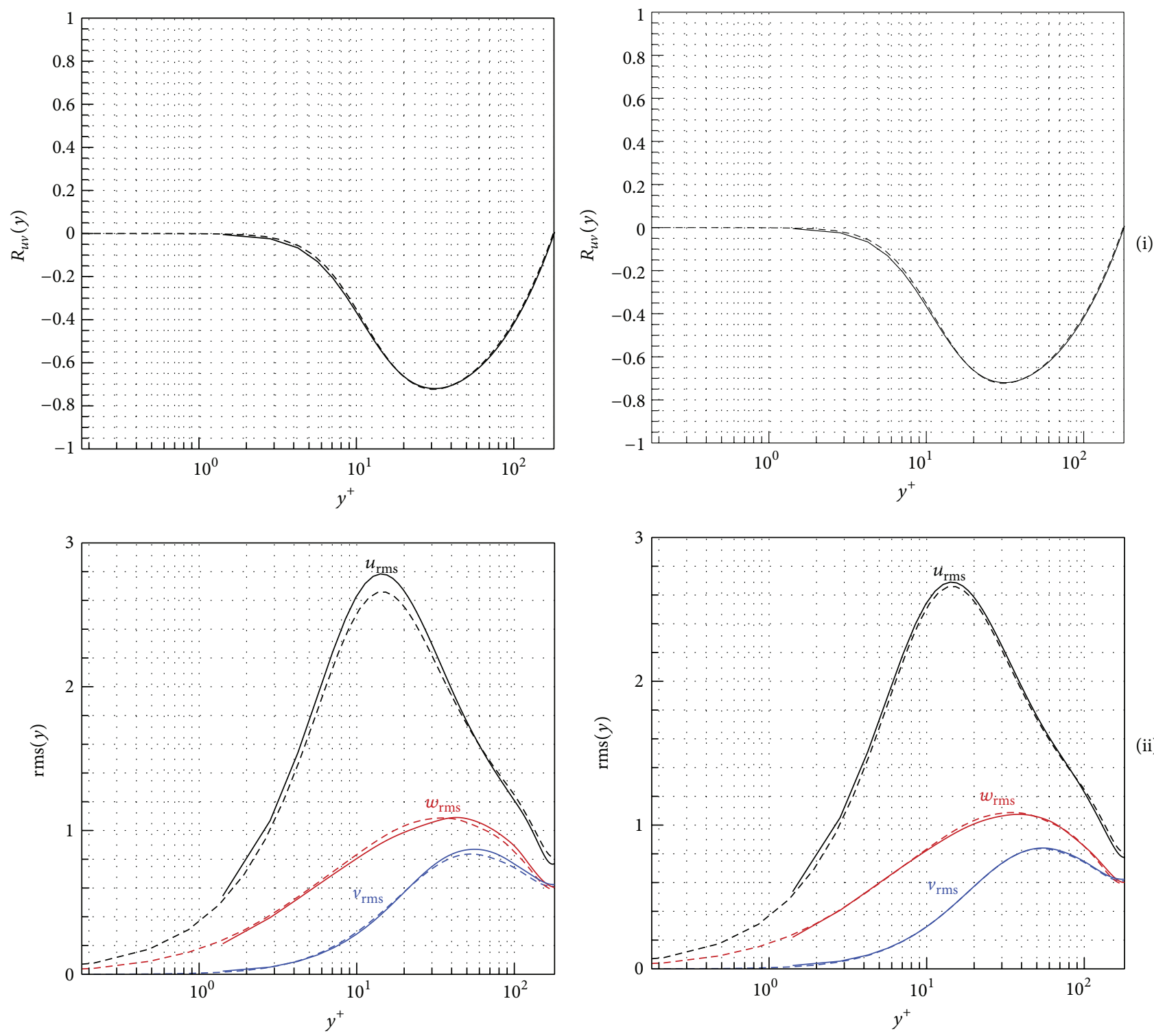

- LBM-DNS

- - - Moser et al. (1999)

- LBM-DNS

- - - Moser et al. (1999)

(a)

(b)

FIGURE 6: The profile of (i) Reynolds stress in the normal $y$-direction and (ii) rms in streamwise $x$-direction, spanwise $z$-direction, and normal $y$-direction at (a) $\alpha_{x}=4, \alpha_{\mathrm{z}}=2$, (b) $\alpha_{x}=8, \alpha_{z}=2$.



FIGURE 7: The strategy of grid refinement of LBM. 


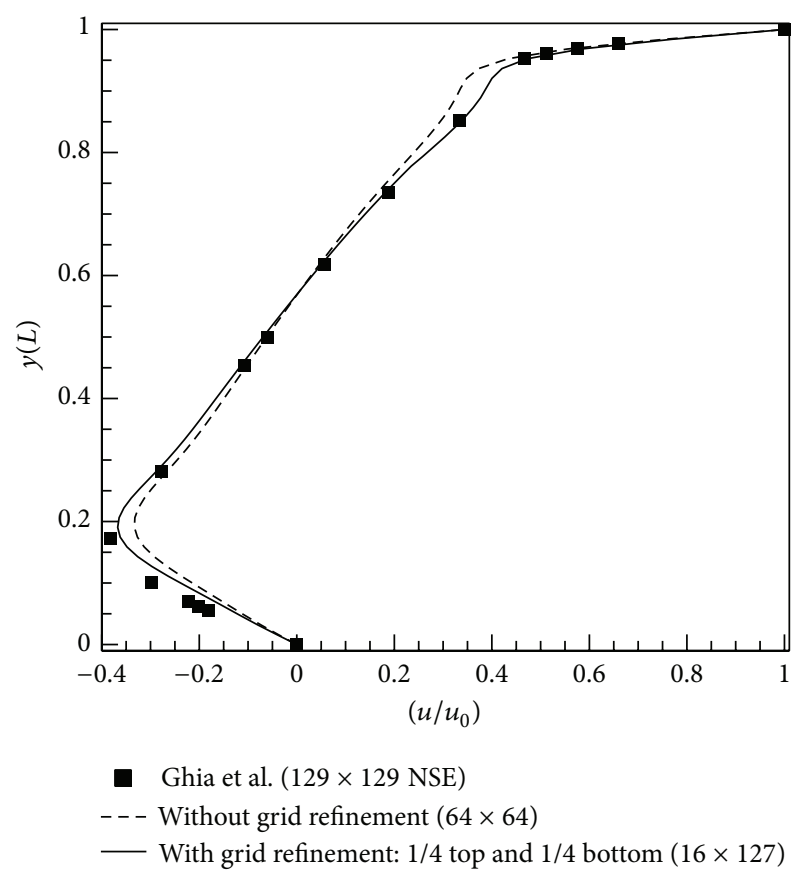

FIGURE 8: The comparison between the NSE results of Ghia et al. [25] and our LBE results with and without using grid refinement on the problem of lid-driven cavity flow: velocity profile on the center line in the normal direction at $\mathrm{Re}=1000$.

same, which means that the used subgrid scale model does not work at such a large grid scale. Considering grid scale and computational cost simultaneously, grids in $1 / 4 \delta$ of top and bottom walls were doubled. Figure 10 shows the mean velocity distribution in wall normal direction at various $C_{s}$ computed in a local grid refinement system, where $\delta / 4$ top and bottom parts adjacent to walls were refined to $512 \times 15 \times$ $127\left(\Delta^{+}=2.82\right)$ and the middle parts are kept at $256 \times 48 \times 64$ $\left(\Delta^{+}=5.64\right)$. As is observed, the subgrid model takes effect and the results get the best match when the value of $C_{s}$ is 0.13 , which is less than the typical value of $C_{s}=0.17$ used in the NS-LES [28].

Figure 11 shows the profile of Reynolds stress in the normal $y$-direction using LBM-LES at $C_{s}=0.13$ for the same local grid refinement system mentioned above. It can be observed that the values deviate LBM-DNS results near the walls, that means the grid is not fine enough to capture the very fine eddies in boundary layer. Also, the SGS model for constant $C_{s}$ is less efficient near walls.

\section{Conclusion}

In the present work, DNS and LES were performed on the wall-bounded flow at $\mathrm{Re}_{\tau}=180$ using lattice Boltzmann method and multiple GPUs. In the DNS, 8 K20M GPUs were adopted. The effect of aspect ratio was verified and the number of grids is $6.7 \times 10^{7}$ for the largest aspect ratio. The results show that although the periodical boundary is used in spanwise and streamwise directions, the dimensions in both directions have effect on the DNS results. For the case of

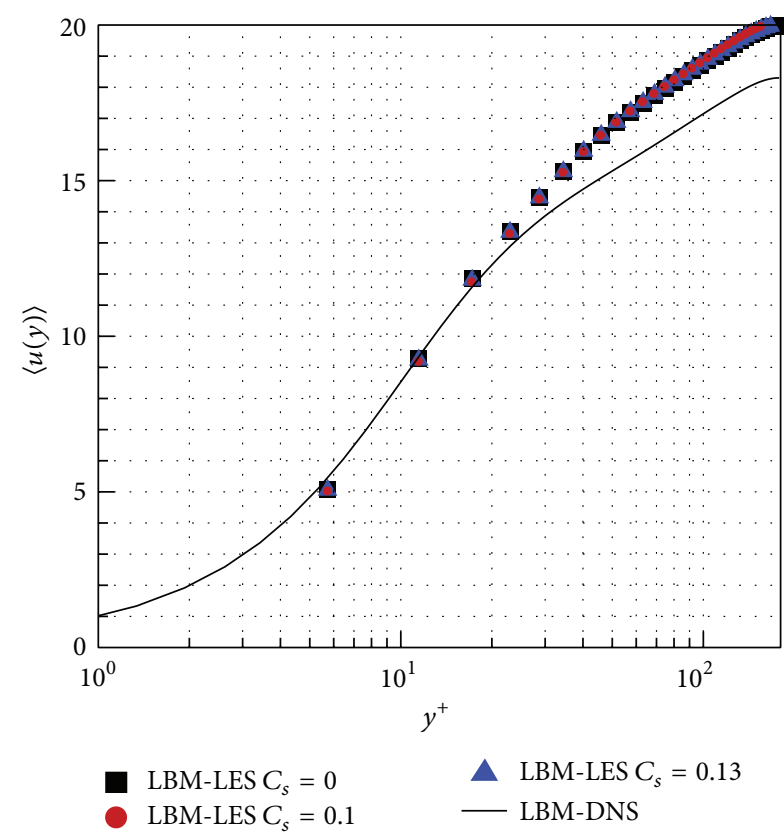

FIGURE 9: The profile of averaged velocity in the normal $y$-direction using LBM-LES at various $C_{s}$ for a grid system $256 \times 64 \times 64\left(\Delta^{+}=\right.$ 5.64).

$\alpha_{x}=8, \alpha_{z}=2$, both the mean velocity and turbulent statistic variables, such as Reynolds stress and velocity fluctuations, agree perfectly with the results of Kim et al. [5]. At the same time, high performance of 2333 MLUPS was obtained. As for the LES, the local grid refinement technique was tested and then used. Using $1.76 \times 10^{6}$ grids and $C_{s}=0.13$, good results on mean values were obtained. However, the turbulent statistic values deviate from LBM-DNS data near the wall. It may suggest that more grids should be used near walls and the dynamic Smagorinsky constant should be utilized to obtain more accurate results. Moreover, multi-GPU, which is of super computing power and matches perfectly with the good parallelism of LBM, presents a surprisingly high performance in this work.

\section{Conflict of Interests}

The authors declare that there is no conflict of interests regarding the publication of this paper.

\section{Acknowledgments}

The authors would like to acknowledge the financial support for this work provided by the National Natural Science Foundation of China (no. 11302165 and 11242010). Also, this research was supported in part by the Japan Society for the Promotion of Science (JSPS) KAKENHI, a Grant-in-Aid for Scientific Research (B) no. 23360046 and a Grant-in-Aid for Young Scientists (B) no. 25870226, and Japan Science and Technology Agency (JST) Core Research of Evolutional Science and Technology (CREST) research programs on "Highly 


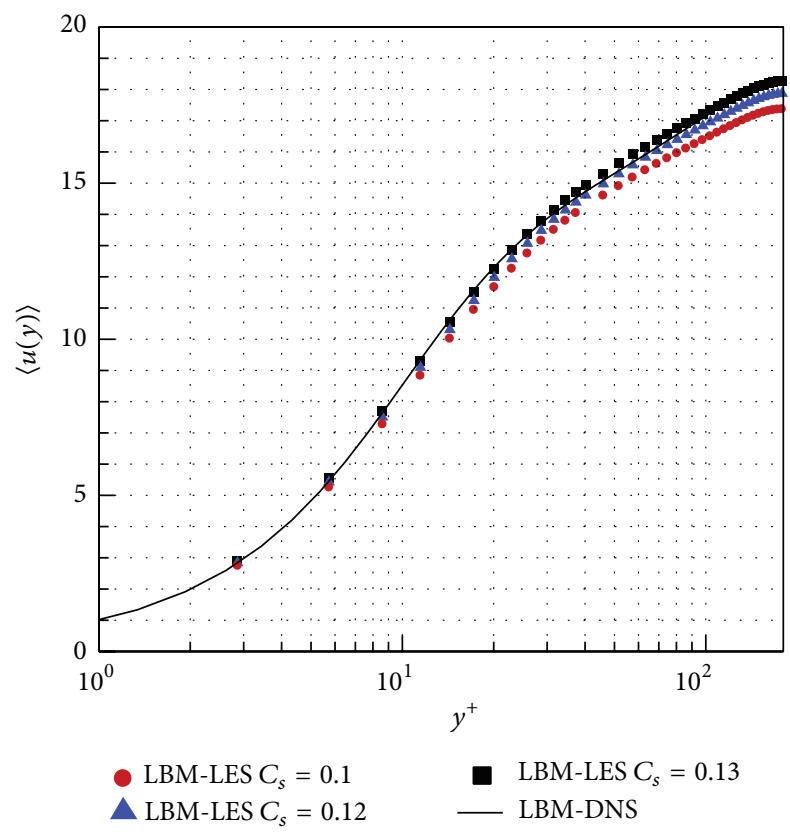

FIGURE 10: The profile of averaged velocity in the normal $y$-direction using LBM-LES at various $C_{s}$ for a local grid refinement system: $\delta / 4$ top and bottom parts adjacent to walls were refined to $512 \times 15 \times 127$ $\left(\Delta^{+}=2.82\right)$ and the middle parts are kept at $256 \times 48 \times 64\left(\Delta^{+}=5.64\right)$.

Productive and High Performance Application Frameworks for Post-Petascale Computing."

\section{References}

[1] S. Chen and G. D. Doolen, "Lattice Boltzmann method for fluid flows," Annual Review of Fluid Mechanics, vol. 30, no. 1, pp. 329364, 1998.

[2] Y. L. He, Y. Wang, and Q. Li, Lattice Boltzmann Method: Theory and Applications, Science Press, Beijing, China, 2009.

[3] H. Yu, S. S. Girimaji, and L.-S. Luo, "DNS and LES of decaying isotropic turbulence with and without frame rotation using lattice Boltzmann method," Journal of Computational Physics, vol. 209, no. 2, pp. 599-616, 2005.

[4] H. Yu, L.-S. Luo, and S. S. Girimaji, "LES of turbulent square jet flow using an MRT lattice Boltzmann model," Computers and Fluids, vol. 35, no. 8-9, pp. 957-965, 2006.

[5] J. Kim, P. Moin, and R. Moser, "Turbulence statistics in fully developed channel flow at low Reynolds number," Journal of Fluid Mechanics, vol. 177, pp. 133-166, 1987.

[6] R. D. Moser, J. Kim, and N. N. Mansour, "Direct numerical simulation of turbulent channel flow up to $\mathrm{Re}=590$," Physics of Fluids, vol. 11, no. 4, pp. 943-945, 1999.

[7] nVIDIA, "NVIDIA CUDA Compute Unified Device Architecture programming Guide Version 2. 0," nVIDIA, 2008.

[8] I. Buck, T. Foley, D. Horn et al., "Brook for GPUs: stream computing on graphics hardware," in Proceedings of the ACM Transactions on Graphics (SIGGRAPH'04), vol. 23, pp. 777-786, August 2004.

[9] J. Krüger and R. Westermann, "Linear algebra operators for GPU implementation of numerical algorithms," ACM Transactions on Graphics, vol. 22, no. 3, pp. 908-916, 2003.

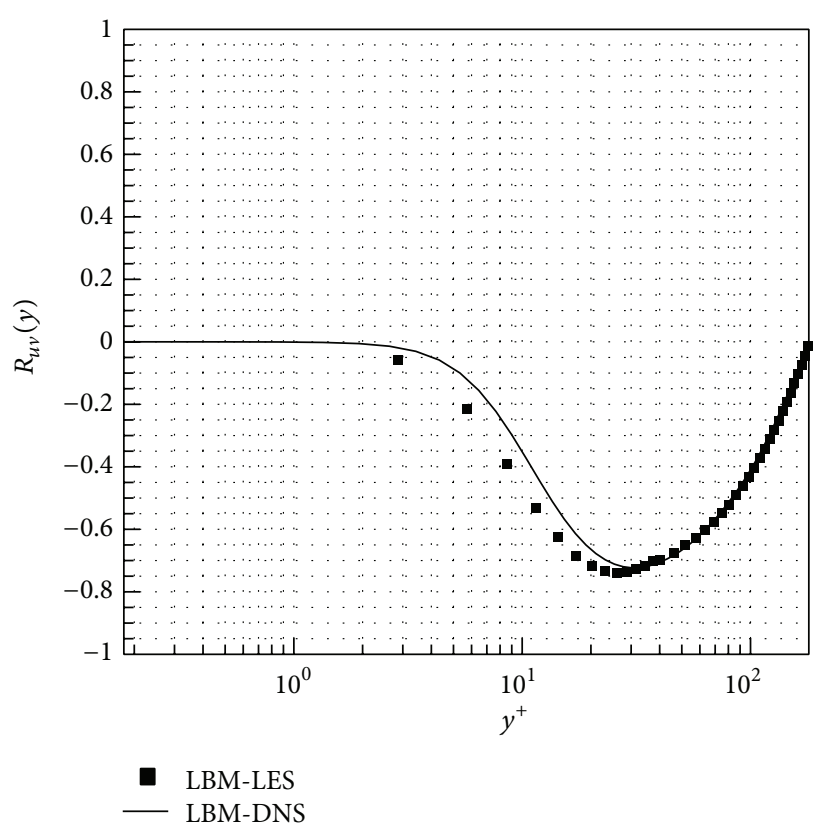

FIGURE 11: The profile of Reynolds stress in the normal $y$-direction using LBM-LES at $C_{s}=0.13$ for a local grid refinement system: $\delta / 4$ top and bottom parts adjacent to walls were refined to $512 \times 15 \times 127$ $\left(\Delta^{+}=2.82\right)$ and the middle parts are kept at $256 \times 48 \times 64\left(\Delta^{+}=5.64\right)$.

[10] S. Ogawa and T. Aoki, "GPU Computing for 2-dimensional incompressible-flow simulation based on multi-grid method," Transactions of JSCES, Article ID 20090021, 2009.

[11] T. Harada, "Smoothed particle hydrodynamics on GPUs", in Proceedings of the Spring Conference on Computer Graphics, pp. 235-241, 2007.

[12] W. Xian and A. Takayuki, "Multi-GPU performance of incompressible flow computation by lattice Boltzmann method on GPU cluster," Parallel Computing, vol. 37, no. 9, pp. 521-535, 2011.

[13] D. Rossinelli, M. Bergdorf, G.-H. Cottet, and P. Koumoutsakos, "GPU accelerated simulations of bluff body flows using vortex particle methods," Journal of Computational Physics, vol. 229, no. 9, pp. 3316-3333, 2010.

[14] http://www.sim.gsic.titech.ac.jp.

[15] T. Shimokawabe, T. Aoki, T. Takaki et al., "Peta-scale phase-field simulation for dendritic solidification on the TSUBAME 2.0 supercomputer," in Proceedings of the International Conference for High Performance Computing, Networking, Storage and Analysis (SC11 '11), New York, NY, USA, November 2011.

[16] T. Shimokawabe, T. Aoki, J. Ishida, K. Kawano, and C. Muroi, "145 TFlops performance on 3990 GPUs of TSUBAME 2.0 supercomputer for an operational weather prediction," in Proceedings of the 11th International Conference on Computational Science (ICCS '11), vol. 4, pp. 1535-1544, June 2011.

[17] X. Wang and T. Aoki, "High performance computation by multi- node GPU cluster- TSUBAME 2. 0 on the air flow in an urban city using lattice Boltzmann method," International Journal of Aerospace and Lightweight Structures, vol. 2, no. 1, pp. 77-86, 2012.

[18] T. Miki, X. Wang, T. Aoki et al., "Patient- specific modeling of pulmonary air flow using GPU cluster for the application in medical practice," Computer Methods in Biomechanics and Biomedical Engineering, vol. 15, no. 7, pp. 771-778, 2012. 
[19] C. Cercignani, Theory and Application of the Boltzmann Equation, Scottish Academic Press, London, UK, 1975.

[20] P. L. Bhatnagar, E. P. Gross, and M. Krook, "A model for collision processes in gases. I. Small amplitude processes in charged and neutral one-component systems," Physical Review, vol. 94, no. 3, pp. 511-525, 1954.

[21] J. A. Somers, "Direct simulation of fluid flow with cellular automata and the lattice-Boltzmann equation," Applied Scientific Research, vol. 51, no. 1-2, pp. 127-133, 1993.

[22] S. Hou, J. Sterling, S. Chen, and G. D. Doolen, "A lattice Boltzmann subgrid model for high Reynolds number flows," in Pattern Formation and Lattice Gas Automata, A. T. Lawniczak and R. Kapral, Eds., vol. 6 of Fields Institute Communications, pp. 151-166, American Mathematical Society, Providence, RI, USA, 1996.

[23] H. Yu, S. S. Girimaji, and L.-S. Luo, "DNS and LES of decaying isotropic turbulence with and without frame rotation using lattice Boltzmann method," Journal of Computational Physics, vol. 209, no. 2, pp. 599-616, 2005.

[24] P. Lammers, K. N. Beronov, R. Volkert, G. Brenner, and F. Durst, "Lattice BGK direct numerical simulation of fully developed turbulence in incompressible plane channel flow," Computers and Fluids, vol. 35, no. 10, pp. 1137-1153, 2006.

[25] U. Ghia, K. N. Ghia, and C. T. Shin, "High-Re solutions for incompressible flow using the Navier-Stokes equations and a multigrid method," Journal of Computational Physics, vol. 48, no. 3, pp. 387-411, 1982.

[26] J. Jimenez and P. Moin, "Minimal flow unit in near-wall turbulence," Journal of Fluid Mechanics, vol. 225, pp. 213-240, 1991.

[27] M. Spasov, D. Rempfer, and P. Mokhasi, "Simulation of a turbulent channel flow with an entropic Lattice Boltzmann method," International Journal for Numerical Methods in Fluids, vol. 60, no. 11, pp. 1241-1258, 2009.

[28] S. B. Pope, Turbulent Flows, Cambridge University Press, Cambridge, UK, 2000.

[29] O. Filippova and D. Hänel, "Boundary-fitting and grid refinement in Lattice-BGK models," in Proceedings of the 7th International Symposium on Computational Fluid Dynamics, pp. 192197, Beijing, China. 




Advances in

Operations Research

mansans

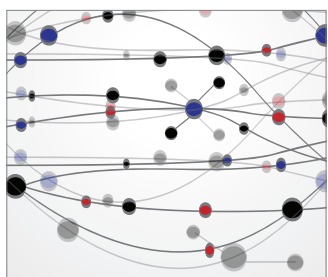

The Scientific World Journal

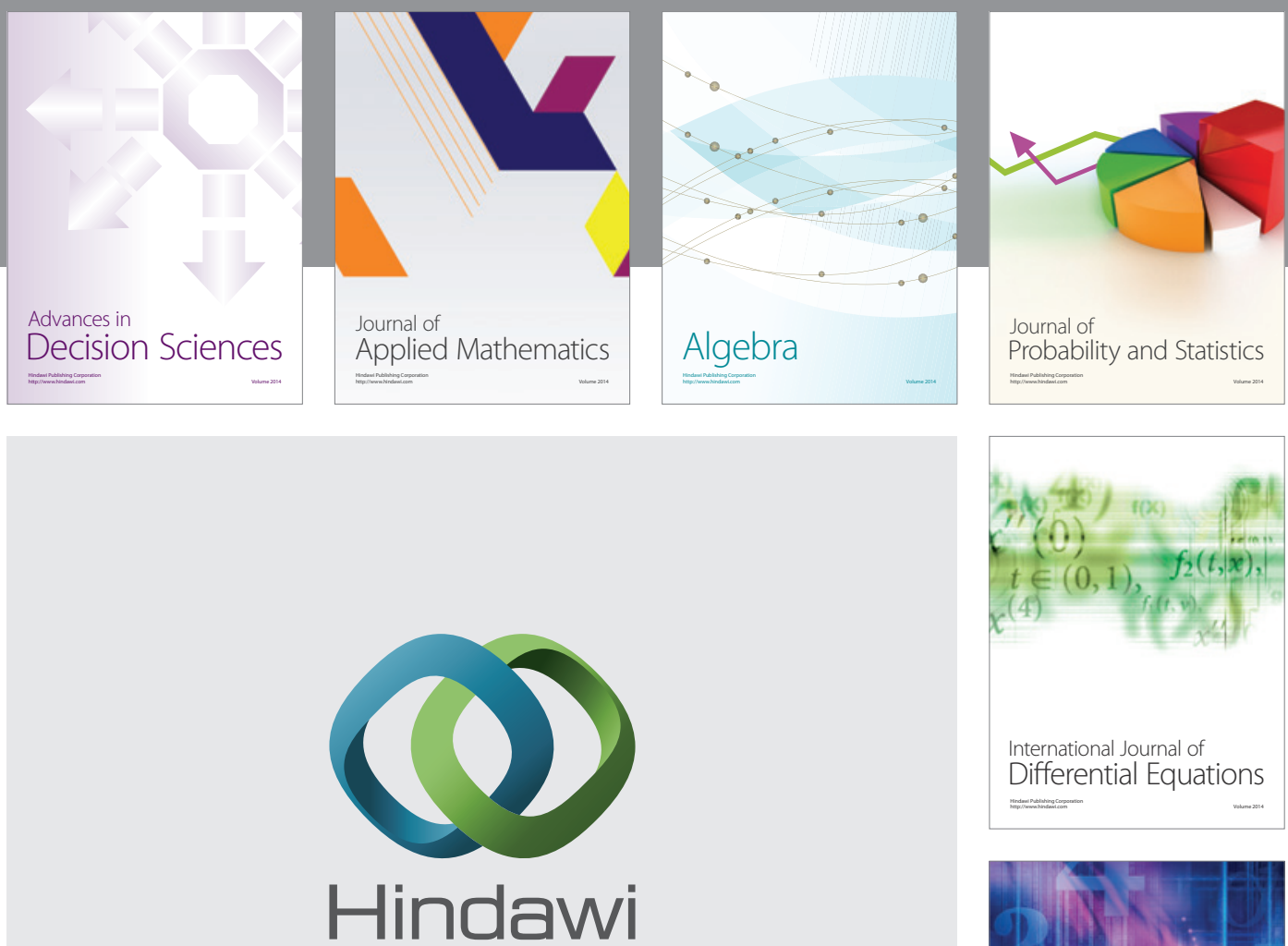

Submit your manuscripts at http://www.hindawi.com
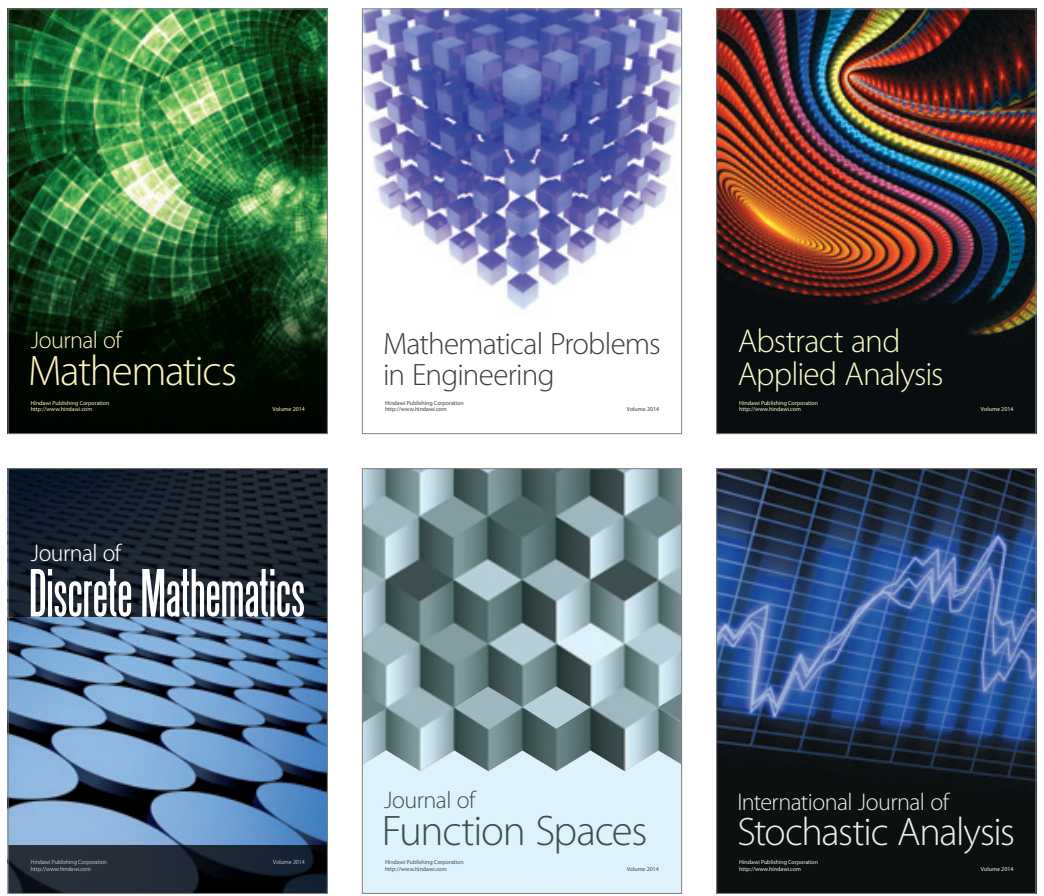

Journal of

Function Spaces

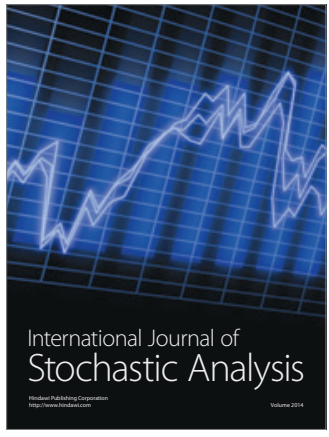


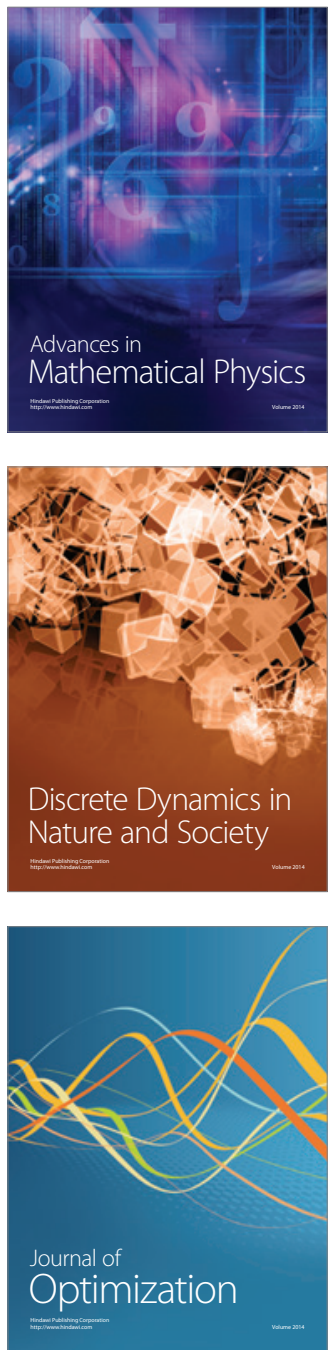